\title{
Ist [der] Stil meßbar?
}

An unbewachten Bahnübergängen warnt in Frankreich ein Schild: $>$ Attention, un train peut en cacher un autre! $<$ Der knappe Titel meiner Ausführungen enthält (zumindest) zwei Fragen: $\gg I s t$ der - namlich <dieser> oder <jener> - Stil meßbar? \& und > Ist Stil - nämlich <an und für sich>bzw. <überhaupt> - meßbar? \&Die Antworten auf diese beiden Fragen lauten verschieden, wenn auch nicht widersprüchlich: $\gg$ Dieser oder jener Stil wird immer meßbarer und $\ll$ Der Stil überhaupt wird immer weniger meßbar $\ll$ bzw. die MeBbarkeit der unterschiedlichen Stile steigt, je leistungsfähiger unsere Apparaturen zur Erfassung und Reproduktion von Strukturen werden. Sie sinkt, je ein-deutiger wir die einmalige Kohärenz des $\gg$ maBgeblichen« Kunstwerkes erschließen.

\section{Zur Begriffsklärung}

In den Kategorien der Ästhetik ist der <generelle> Begriff / Stil/ höher angesiedelt als der <spezielle> Begriff /Sprachstil/ und folgerichtig auch als die partikuläreren Begriffe /Brief-/, /Roman-/, / Traktat-/ oder /Vortragsstil/. In den Kategorien einer umfassenden, weil Logisches, Psychologisches und Pragmatisches einschließenden Grammatik hat denn auch Stilistisches seinen Platz. Der Grammatiker darf sich deshalb der stilistischen Verantwortlichkeit nicht entledigen und etwa den <Stil an und für sich> sozio-linguistisch und psycho-linguistisch als eine unstete Menge von $\gg$ repressiven Konventionen $\ll b z w$, von $\gg$ Kollektiv-Subjektivismen $\ll$ betrachten, deren Analyse sich nur an Außersprachlichem zu orientieren hätte, als ginge es dabei lediglich um $\gg$ Soziokulturelles $\ll$, etwa Gewöhnung, Intoleranz, Prestige, Absonderung, Mode, ja Politik, wobei eine multistilistische [sic] Begabung besonders zu würdigen bzw. mit einem Literaturpreis auszuzeichnen wäre.

\section{Was hat [der] Stil für Attribute?}

Zur methodischen Naivität gehört die Erfassung der <Prädikate>, mit denen der Stil für gewöhnlich von den Kritikern bedacht wird, zum Beispiel akademisch, ausgefeilt, barbarisch, blühend, dunkel, eigenwillig, einfach, elegant, emphatisch, energisch, episch, farblos, gehoben, gepflegt und ungepflegt, geblümt, gewählt, geschliffen, geschminkt und ungeschminkt, geschwollen, harmonisch, hochtrabend, impressionistisch und expressionistisch, kapriziös, klassisch, konzis, langatmig, langstielig, langweilig, lebendig, lichtecht, morbid, mühsam, naiv, ob- 
skur, pittoresk, preziös, quirlig, roh, rhetorisch, romantisch, schlampig, schlecht, schlicht, schwungvoll, streng, stümperhaft, tänzelnd, trocken, überschwenglich, ungeziert, verschleiert und unverschleiert, verschwommen, weitschweifig, und - vielleicht etwas meBbarer? - wortkarg und wortreich, sowie, um es nicht vergessen zu haben: künstlich, natürlich, stilvoll und ... stillos.

Das auf CD-ROM befragte 'Trésor de la Langue Française' brachte das Doppelte zum Vorschein, u.a. déchiré, effacé, épiscopal, lapidaire, miroitant, nerveux, opulent, perspicace, rocailleux, soutenu, tourmenté und villageois, die als nachgestellte Adjektive klassifizierende Ansprüche anmelden, während beau, fier, fort, foutu, grand und haut immer vorangestellt und somit nur zur - übrigens laschen - Bewertung gebraucht werden; nur vier Adjektive erscheinen in beiden Stellungen bzw. Funktionen, nämlich abominable, excellent, long und mauvais.

Komposita wie Amtsstil, Barockstil, Biedermeierstil, Funktionärsstil, Jugendstil, Kanzleistil, Renaissancestil und Übergangsstil oder Stilblüte, Stilbruch, Stilebene, Stilelement, Stilempfinden, Stilfehler, Stilfigur, Stillehre, Stilmittel und Stilmöbel sind jedem geläufig; desgleichen die Stilnote - bei den Olympischen Spielen für eine immer entscheidende und oft bemängelte <Messung> und das Stilwörterbuch als <phraseologisches Wörterbuch, das die korrekte Verwendung der Wörter im Satz darstellen soll>.

Der französische Terminus „stylométrie” bezeichnet seit einem Jahrhundert die <angewandte statistische Stilkunde> als philologische Methode zur Erforschung der Chronologie der Dialoge Platons, um die Jahrhundertwende, und neuerdings zur Echtheitsprüfung aristotelischer (?) Fragmente. Das Programm des Pariser internationalen Kolloquiums Consensus ex machina? (Sorbonne, April 1984) verzeichnet zwei Beiträge zur jüngsten Entwicklung dieser Disziplin, bis an die Schwelle forensischer Verwendung, wie etwa aus den jüngsten Arbeiten von Christian Rutten (Lüttich) und Alfred Noé (Wien) hervorgeht.

\section{Analoga aus der bildenden Kunst}

Vorstellungen von Stil-Arten und Stil-Qualitäten hat nicht nur der literarische Stilkundler. Es sind vermutlich zum Teil nicht immer die gleichen - auch nicht oder gerade nicht bei Zeitgenossen. Dennoch lassen sich in der bildenden Kunst sozusagen formale Atome und Moleküle auflisten, wie dies etwa in der mehrbändigen Sammlung $\gg L a$ Grammaire des formes et des styles $\ll$ im Schweizer Verlag Office du Livre (Fribourg) durchgeführt wurde. Während der Literaturhistoriker in der Regel nur 
zwei oder drei Jahrhunderte überblickt, überbrückt der komparatistische Ansatz der Kunstgeschichte Jahrtausende.

Anhand besonderer Themen, etwa der Darstellung des Pferdes (etwa in Les Chevaux - Un si long compagnonnage von Roger-Louis Thomas, Paris Hatier 1993, und im Kapitel Le Cheval en Chine et ses représentations, von Hubert Delahaye, $\mathrm{CdF}$, in Le petit livre du cheval en Chine, Paris Caracole, nicht datiert) zeigt sich, daB die abstraktesten Formen immer wieder zu Abkehr und Umkehr AnlaB boten, und daß Wechsel und Erneuerung nicht weniger als rituale Schulbildung zur Stilgeschichte gehören, welche sich demnach nicht dichotomisch bzw. einfach in $>$ klassisch $\ll$ vs. $>$ postklassisch $\ll$ oder $\gg$ romantisch $\ll$ vs. $\gg$ vorromantisch $\ll$ bzw. $\gg$ statisch $\ll$ vs. $\gg$ dynamisch $\ll$ oder $\gg$ objektiv $\ll$ vs. >subjektive einteilen läßt. Das Bewegte gehört sozusagen zum Stetigen.

Eine vornehmlich aus dem Buch von Roger-Louis Thomas geschöpfte Auswahl von dreiunddreißig Darstellungen des Pferdes, von der Höhlenmalerei von Lascaux bis zu Théodore Géricault, von altchinesischen Schattenrissen bis Velasquez, von Persien bis zur mittelalterlichen Kaiserchronik, von Vanini bis zu Seurat, von Stubbs bis zu Magritte, vom Cheval bleu von Paul Gauguin bis zur Carriole du Père Juniet vom Douanier Rousseau, folgte einem Wink Platons, der, bevor er eine Definition des Sophisten geben wollte, zunächst irgendetwas definierte, was jeder kannte und von dem jeder wuBte, was es war, und deswegen die Brauchbarkeit der Definitionsprozedur beurteilen konnte, nämlich die Angelfscherei. Wer nicht wüBte, was ein Pferd ist, erführe zum Beispiel aus der Enzyklopädie, daB $\gg$ die weiblichen Tiere nach etwa elfmonatiger Tracht (meist) ein Junges (Fohlen) gebären $\ll$, was ihm zugleich zeigt, was unter / enzyklopädischem Stil/zu verstehen ist. Bei der Betrachtung dieser Bilder sollte man die Mahnung eines französischen Malers nicht überhören, daß sich die Malerei, wie die Literatur, nicht eigentlich an das Auge wendet, $\rightarrow \dot{a}$ l'oeil $\ll$, sondern, wie das auch immer zu übersetzen ist, $\gg \dot{a}$ l'esprit<. Von dieser kurzen, klassischen Kunstfabel hieße die lange, ebenfalls klassische Moral, daßder $\mathbf{S}$ til in der $k$ oh ärenten und stabilisierten Wahl best eht. Auch der Einwand, daB Pferd nicht einfach Pferd, sondern Reit- oder Zugpferd, Renn- oder Arbeitspferd sei, würde der Sache nicht schaden, da $\gg P$ Perderassen $\ll$ wie Stile durch »stabil gezüchtete Merkmale $\ll$ geschaffen werden.

In der bildenden Kunst wird nicht nur vom Stil des einzelnen Werkes oder Künstlers gesprochen, sondern, mit nicht weniger Prägnanz, vom Stil von Schulen, Epochen und Kulturen. Daß eine Stilfibel nur eine engere Grammatik, eine Art 》grammaire choisie«, sein kann, ist eine 
Lappalie. Wie sieht es am anderen Ende der Kette aus: kann eine allgemeine und umfassende Grammatik ohne Stil auskommen bzw. ihre Zuständigkeit aufgeben, wenn es um Ästhetik geht, weil sie sich zur Stilistik so verhielte, wie Pflicht zu Kür? In seinen Grammatischen Gesprächen überlieb Klopstock zwar einen entscheidenen Part der $\gg K u ̈ r \ll$, aber es war sozusagen ein $\gg$ innergrammatischer $<$.

Meine These lautet denn auch, daB Grammatik ohne Stilistik nicht denkbarer und machbarer ist als ein Text ohne Form, als eine Sprachlehre ohne Logik und ohne Pragmatik. Wollte man weiterhin wie gewohnt die Stilistik aus der Grammatik aussiedeln, entstünde ein Niemandsland zwischen einem Katalog von prinzipiellen <Geboten > und mehr oder minder scharfen <Verboten>. Das Prinzip, nach dem alles erlaubt ist, was nicht verboten ist, genügt nicht, um sich einen Stil zu schaffen. Als Merkmale zu England, Preußen, Rußland und Österreich in der napoleonischen Zeit der ersten Belege zu stylométrie, allerdings als $\gg$ Säulenmessung $\ll$, soll für den $\gg$ offentlichen Lebensstilk gegolten haben, es sei jeweils $\gg$ alles erlaubt gewesen, was nicht verboten war; alles verboten, was nicht erlaubt; alles verboten, was erlaubt; alles erlaubt, was verboten war. \& Mit solchen Verfassungen lieBe sich keine Stillehre schreiben. Wegen dieser Lücke in den Sprachlehren haben die Stilpädagogen zwei andere Wege bevorzugt: die korrigierte Spontaneität und das immer empfehlenswerte Abschreiben und Auswendiglernen von Mustertexten, womöglich aus einer Quelle: etwa aus den $\gg$ Wahlverwandtschaften $\ll$, die sich Thomas Mann zu diesem Behufe ausgesucht haben soll.

\section{Stilfragen als grammatische Fragen}

Die Grammatik lehrt zum Beispiel, daß ein Satz beliebig lang sein kann und daß jeder Satz erweitert werden kann. Sie sagt aber nicht, wann welcher Satz wie lange sein darf.

Die Grammatik lehrt zum Beispiel, daß für Nomina Pronomina eingesetzt werden können. Davon abgesehen, daß sie bestimmte Verbotslisten nicht motiviert und nicht zu merken scheint, daß die Unmöglichkeit, ein im Rhema stehendes Substantiv anaphorisch aufzugreifen, nicht als <Ausnahme> zu katalogisieren, sondern als < Regel> zu verstehen ist, sagt sie nicht, wann ein Pronomen eingesetzt werden darf oder soll, und warum.

Die Grammatik lehrt zum Beispiel, daß im unabhängigen sogenannten Kernsatz ein beliebiges Satzglied das Vorfeld besetzen kann, was je nachdem in einem Falle zur geraden und in allen anderen Fällen zur ungeraden Wortstellung führt. Sie bleibt aber die Antwort schuldig auf die 
nicht nur für das <Deutsche als Fremdsprache>, sondern auch für das <Deutsche als Muttersprache> wichtigen Fragen: warum so, warum anders, warum überhaupt?

Das gilt nicht nur für ästhetische Erwartungen, sondern auch für logische Belange. Wenn die Grammatik im Kapitel <Satzbaupläne> lehrt, daß dem <Satzgegenstand > der <Prädikatsverband> gegenübersteht, ohne zu sagen, daB sowohl das Akkusativobjekt wie das Nominativsubjekt zu diesem oder jenem gehören oder auch nicht gehören können, wird sie weder eine ordentliche Syntax axiomatisieren noch die Brücke zur Textgrammatik schlagen können. Insbesondere wird sie nicht sagen können, welche Bereiche die Logikder Stilistikfrei-

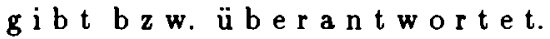

Wenn sie dagegen - schließlich? - meint, die Stilistik sei für alle Verschränkungen, Verwindungen und Verrenkungen zwischen der postulierten <Tiefenstruktur> und den registrierten <Oberfiächenstrukturen> zuständig, sagt sie nicht zu wenig, sondern - schlimmer - z u vi el.

\section{Zur eigentlichen $\gg$ Stilometrie $\ll$}

Die Fragen, ob <der Stil > meßbar ist und ob <Stil überhaupt $>$ meßbar ist, gehen schon deswegen den Grammatiker an, weil alle Stilmittel Sprachmittel und mit geeigneten Mitteln <meßbar> im Sinne von <abzählbar> sind.

Vor bald vierzig Jahren, als bei der Neuauflage des $\gg$ Wegweisers zum treffenden Ausdruck von Wehrle Hans Eggers in Hamburg von den Kastenzetteln zu den Lochkarten überging, habe ich den Brief $\gg$ holleritiert $\ll$, in welchem im Juni 1652 Pascal seine Rechenmaschine der Königin Christine von Schweden erklärte. Inzwischen sind meine Hamburger Lochkarten fast zu Staub verfallen. Unter den geretteten befinden sich diejenigen, die folgende Stelle betreffen: $\gg$ Cet ouvrage, $\mathrm{Ma}$ dame, est une machine pour faire les règles d'arithmétique sans plume et sans jetons. Votre Majesté n'ignore pas la peine et le temps que coûtent les productions nouvelles, surtout lorsque les inventeurs les veulent porter eux-mêmes à la dernière perfection; c'est pourquoi il serait inutile de dire combien il y a que je travaille à celle-ci; et je ne peux mieux l'exprimer qu'en disant que je m'y suis attaché avec autant d'ardeur que si j'eusse prévu qu'elle devait paraître un jour devant une si auguste personne $\ll$ - verdeutscht und postmodernisiert: $\gg$ Dieser Computer ist eine Maschine, die ohne Schreibzeug und Abakus die Grundrechenarten ausführen kann. Wieviel Mühe und Zeit ein perfekter Prototyp kostet, ist Ihnen hinlänglich bekannt. Ich hätte nicht mehr Aufmerk- 
samkeit und Ausdauer daran verwenden können, wenn ich geahnt hätte, $\mathrm{daB}$ dieser mechanische Rechner eines Tages Ihrer lieblichen Hoheit vorgestellt werden dürfte. $\ll$ Aus der gleichen Gründerzeit stammen vergilbte Formulare zur Auszählung von bestimmten Text-Merkmalen wie Satzlänge, Anapher- und Katapher-Gebrauch, Häufung von attributiv verwendeten Adjektiven, Verhältnis von parataktischen und hypotaktischen Strukturen usw. Frequenz-Untersuchungen zum Wortschatz habe ich damals aus zwei Gründen vernachlässigt: einerseits gab es schon anderswo großangelegte und reichdotierte Untersuchungen zur stilistischen Lexikologie bzw. $>$ Lexikometrie<; andererseits verfügte ich über keinerlei sogenannten Projektgelder und hatte mir die stilometrischen Experimente sozusagen als analytische Fingerübungen ausgedacht, bei denen meinen zur Aufmerksamkeit gezwungenen Studenten fast nebenbei allerhand auf- und einfallen könnte. Damals gab es weder Projektgelder noch Beiräte: die privat zu begleichende Monatsmiete der Lochmaschine betrug $5 \mathrm{DM}$.

Die damalige schlechthin experimentelle Methode halte ich heute weiterhin bzw. wiederum für sinnvoll, denn es ist gar nicht so leicht, zu wissen, w o r a n Originalität und Qualität eines Stils liegen. Nur könnte man heute durch Computereinsatz viel mehr Aspekte berücksichtigen und etliche nicht intuitive Frequenzwerte, Abweichungen und Schwankungen schneller berechnen. Die angewandte Statistik - wenn dies kein Pleonasmus ist - hat seit den Untersuchungen zu den Nachtwachen des Bonaventura große, wenn auch noch nicht abgeschlossene Fortschritte gemacht, welche insgesamt die eingangs formulierte Ansicht nicht <falsifizieren>: $>$ Meßbar ist dieser oder jener Stil sehr wohl, Stil überhaupt dagegen nichtK.

\section{Zu den Stil- $\gg$ Gestalten $\ll$}

Aus verschiedenen Zählungen diverser vermuteter Stilmerkmale hatten wir einige ausgewählt, bei denen sich bei Textmengen im Umfang von 50.000 Wörtern im Kontinuum - erprobte und gutausgerüstete Statistiker kommen längst mit weniger aus - bestimmte Konstanten errechnen ließen, die je nach dem Autor für ein Buch, für eine Schaffensperiode oder für das Gesamtwerk brauchbar waren. Eine Schilderung der Stilogramme befindet sich in der Vergleichenden Grammatik, FranzösischDeutsch, Band 2, L'économie de la langue et le jeu de la parole, B.I. 1984, 》Läßt sich Stil messen, beschreiben, nachahmen?《, (S. 614-626)

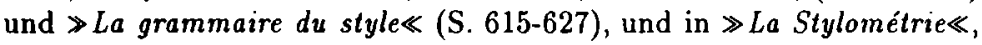
Cahiers du C.R.A.L. n², Nancy 1966 (S. 36-40), von Pierre Girault in 
La Stylistique übernommen, Initiation à la Linguistique A1, Klincksieck Paris 1970 (S. 214-221).

DaB am Ende nur acht Angaben selegiert wurden, lag zum Teil natürlich daran, daß gerade diese charakteristisch zu sein schienen. Dieser Minimalismus hatte auch mit der instinktiven Ahnung etwas zu tun, daB der Stilsich nicht einfach auf eine Anhäufung von Einzelformen zurückführen $l \ddot{a} B t$, sondern irgendwie einer $>$ Gestalt<entspricht, nat ürlich in dem Sinne, in dem die Wahrnehmungslehrevon/Gestalt/spricht. Die Selektion von nur acht Werten erleichterte die Darstellung der verschiedenen Messungen als Dimensionen. Um Sinnfälliges und Augenfälliges zu vereinen, übersetzten wir diese Dimensionen in Strahlen. Je nach ausgezählten Daten (etwa die Zahl der Adjektive in attributischer Funktion und die Zahl der Satzartikel /daß/ im Nominativ) waren Werte zu erhalten, deren Proportion zueinander den Vergleich unnötig erschwerte: eine Schwankung von $2 \%$ ist bei einem Mittelwert von $18 \%$ fast uninteressant, während sie bei einem Mittelwert von $1 \%$ ganz enorm bzw. abnorm wäre. Infolgedessen wurde jeder Dimension ein eigener Maßstab zugeordnet. Der Schnittpunkt der Strahlen mit dem Kreis bestimmte, über einen errechneten Mittelwert, die jeweilige Einheit bzw. den Kurswert.

Die Umwandlung von ursprünglich aus Qualitativem bezogenem Quantitativem in abermals Qualitatives beruhte $\mathrm{zwar}$ auf Konventionen, erleichterte aber die Charakterisierung von Stil-Profilen und -Profilfamilien. Das Verbinden der auf den verschiedenen Skalen eingetragenen Größen und das Schraffieren der so entstandenen Figuren zu Stilgestalten war dann wirklich kein Kunststück mehr. Der Unterschied zwischen den $<$ Gestalten> bzw. <Stilogrammen> entsprach der empfundenen Differenz und fand sich denn auch in Parodien wieder. Darauf, da $B$ die Plagiatoren zünftige angewandte empirische, wenn auch intuitive $<$ Stilometrie> betreiben, ist hier nicht weiter einzugehen, zumal im Anhang das 'Preisrätsel' Anlaß zu einigen Bemerkungen zum Themenkreis Parodie und Fälschung gibt.

Die wissenschafliche Stilometrie hat sich natürlich nicht um die Übersetzung ihrer Messungen in solche $\gg$ Stilogramme $\ll$ bemüht, sondern, wie jede Feinst-Statistik, jenseits aller visuellen 'Gestalten', Konstanten höheren Grades errechnet, $\gg$ Derivate $\ll$, wie bereits in der 1964 vorgelegten und von C.F. v. Weizsäcker und D. Gerhardt betreuten Dissertation von Joachim Thiele nachzulesen ist: Verfahren der statistischen Aesthetik (VVG Hamburg-Altona 1966). Dennoch dürfte gerade das Intuitive und Grobschlächtige der primitiven $\gg$ Stilogramme $\ll$ richtig gelegen haben, 
denn die detaillierten Einzeldaten führen nur dann zu den gesuchten holistischen $\gg$ Gestalten $\ll$, wenn der Statistiker sie rechnerisch so betreut, daB ihre $>$ Streuungen $<$ sich in $\gg$ Kraftlinien $<$ verwandeln, wie sich Eisenfeilspäne auf dem Blatt Papier, das der Lehrer über den Magneten hält, zu eindeutigen Figuren sammeln.

\section{Was nicht meßbar ist}

Abzählen und messen lassen sich offenbar formale Einzeldaten, <dieser oder jener Stil $>$, in Wortlaut meiner Frage also $>\mathrm{d}$ e r S t il «. Wie steht es mit dem Stil überhaupt? Kurzum: $>$ Ist Stil meßbar?

Diesen oder jenen meßbaren Stil nannte Goethe nicht $\gg$ Stil $\ll$, sondern $>$ Manier $<$. Dem Vierzigjährigen lag daran, $>$ das Wort Stil in den höchsten Ehren zu halten, damit uns ein Ausdruck übrig bleibe, um den höchsten Punkt zu erreichen, welchen die Kunst je erreicht hat und je erreichen kann. Diesen Grad auch nur zu erkennen, ist schon eine große Glückseligkeit.\& Wie die Ethik ist auch die Ästhetik in der Aufklärung problematisiert worden. Die Problematik des Stils wurde schneller ausgelebt als ausgedacht, wenn man auf so komplexe Umwälzungen einen so flüchtigen Blick werfen kann.

Als Georges Louis Leclerc, Graf von Buffon, genau ein halbes Jahrhundert vor 1789, im Alter von 32 Jahren in die Académie française aufgenommen wurde, meinte er mit seinem Satz, $\gg$ le style, c'est l'homme même«, weder den Stil überhaupt, noch den Menschen überhaupt, sondern eben, für beide, diesen oder jenen. Buffon war ein Naturalist. Wie hätte er vor lauter Individualität und Originalität der Ausführungen die Zugehörigkeit zu Art und Wesen vergessen können?

Der nur 5 Jahre jüngere Diderot vernahm hinter Senecas (wahrscheinlich des Vaters und des Sohns) $\gg$ talis oratio qualis vita《 die Forderung, die Universalität in der Persönlichkeit zu erreichen. Es wurde ihm bekanntlich vorgeworfen, von der Literatur $\gg$ Moral $\ll$ zu erwarten bzw. zu verlangen. Wie die meisten Denker der Zeit war er von der Malerei fasziniert. Wenn er so von Chardin begeistert war, könnte es an einer solchen <objektiven Relativität> gelegen haben. 15 Jahre nach Diderots Tod, 25 Jahre nach dem Abschluß der Encyclopédie, 10 Jahre nach der Pariser Wende, 7 Jahre nach der Schlacht von Valmy, noch während der Vorbereitung des Staatsstreiches, von dem der dreißigjährige Napoleon - er war 20 Jahre jünger als Goethe - träumt, und derenthalben er in Ägypten sein siegreiches, aber aussichtsloses Expeditions-Korps verläßt, vertieft Goethe diesen <objektiven Relativismus> Diderots: die beschreibbare und meßbare Form allein kann die erstrebte Vollkommen- 
heit der Substanz nicht garantieren. Er schreibt: $\gg$ Das Resultat einer echten Methode nennt man Stil, im Gegensatz der Manier. Der Stil erhebt das Individuum zum höchsten Punkt, den die Gattung zu erreichen fähig ist; deswegen nähern sich alle großen Künstler einander in ihren besten Werken. [...] Dieses gilt so gut vom Sittlichen als vom Künstlichen; denn da alle Handlungen des Menschen aus e i n e $\mathrm{r}$ Quelle kommen, so gleichen sie sich auch in allen ihren Ableitungen. $\ll$ Offenbar ist der $\gg$ Stil $\ll$ das Erstrebte, wenn nicht Unerreichbare, und steht deshalb, wie alles Ideale, außerhalb der Meßbarkeit. Eben im Gegensatz zur bloßen $\gg$ Manier $\ll$. DaB Goethe diese $\gg$ Manier $\ll$ jedoch nicht verachtete, etwa im abwertenden Sinne von $\gg$ Manierismus $\ll$, geht schon daraus hervor, daB er die $\gg$ Manier $\ll$ nicht in eine binäre Opposition zum $\gg$ Stil $\ll$ stellt. Es gebe ein Drittes, die $\gg$ Nachahmung $\ll$, gewissermaßen eine Vorstufe, wie aus einem Vergleich der Verhältnisse dieser drei Formen zur Natur erhellt: $\gg$ Wie die einfache Nachahmung auf dem ruhigen Dasein und einer liebevollen Gegenwart beruhet, die Manier eine Erscheinung mit einem leichten, fähigen Gemüt ergreift, so ruht der Stil auf den tiefsten Grundfesten der Erkenntnis, auf dem Wesen der Dinge, insofern es uns erlaubt ist, es in sichtbaren und greiflichen Gestalten zu erkennen $\ll$. Zehn Jahre später kommt er darauf zurück. Nachdem er, in Anlehnung an Diderot, wiederholt hat, daß der Stil das Individuum zur edelsten Humanität führt, schreibt er: $\gg$ Die Manier hingegen in $\mathrm{d}$ i id u a $\mathrm{l}$ is i e t wenn man so sagen darf, noch das I $\mathrm{n} d \mathrm{i} v \mathrm{i} \mathrm{d} u \mathrm{u} m$ Der Mensch, der seinen Trieben und Neigungen unaufhaltsam nachhängt, entfernt sich immer mehr von der Einheit des Ganzen, ja sogar von denen, die ihm allenfalls noch ähnlich sein könnten; er macht keine Ansprüche an die $\mathrm{M}$ e $\mathrm{ns} \mathrm{ch}$ h i $\mathrm{t}$ und so trennt er sich von den $\mathrm{M}$ e $\mathrm{n} \mathrm{s} \mathrm{ch} \mathrm{n} . \ll$ Drei Jahre, nachdem Napoleon Bonaparte im Alter von dreiundfünfzig Jahren gestorben war, sagt der fünfundsiebzigjährige Goethe sozusagen abschließend zu Eckermann: \Im ganzen ist der Stil des Schriftstellers ein treuer Abdruck seines Innern: will jemand einen klaren Stil schreiben, so sei es ihm zuvor klar in seiner Seele: und will jemand einen großartigen Stil schreiben, so habe er einen großartigen Charaker. $\ll$ Werden die Eigenschaften /klar/ und /großartig/ in der klassischen Stilistik nicht als geeichte Prädikate vergeben?

Der Altmeister Jean Fourquet [dessen beim Goethe-Institut erhältliches Video-Porträt in Mannheim vorgeführt wurde] machte mich darauf aufmerksam, daB auch /Nachahmung/ etymologisch mit /Meßbarkeit/ zu tun hat, und daß sogar, kurioserweise, das nomen Ohm zu omen wurde. Im Rahmen seiner 1922 in Weimar und in Dresden geschriebenen Diplomarbeit über Anton Raphael Mengs hat sich Jean Fourquet übrigens 
auch mit der von Goethe schon vor der Italienischen Reise praktizierten Unterscheidung $\mathrm{zwischen} \gg$ Stil $\ll$ und $\gg$ Manier $\ll$ befaßt.

In der französischen Geistesgeschichte ist übrigens die Humanisierung der Objektivität, wie man diesen $\gg$ objektiven Relativismus « auch nennen kann, bereits in den $\gg$ Essais $<$ von Michel de Montaigne zu erkennen.

\section{Lösung des Paradoxes?}

Hinsichtlich der Meßbarkeit scheint aber diese Akzeption von /Stil/ aus der Perspektive des ERGON in die der ENERGEIA gerückt zu sein. Wenn es nur um die - mit Verlaub pleonastisch zu insistieren $-\gg$ formale Gestalk geht, ist man versucht, zur Ergon-Perspektive zurückzukehren, um sie nicht mehr zu verlassen, zumal nach der nicht zu Ende gedachten Problematik der 》objektiven Relativität《 diejenige der 》subjektiven Relativität《 alias des $\gg$ Nihilismus $\ll$ getreten ist - mit anderen Worten: nicht die von einigen sorgenvoll erhoffte Umwertung aller Werte, sondern ihre beschleunigte $A b$ wertung. Die $\gg$ absolute Unverbindlicheit $\ll$

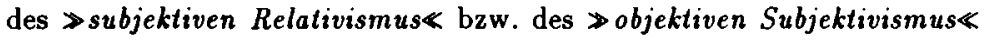
hat jedoch ein paar schwache Nähte, wie der Stil-Wechsel (?) im ModeGeschäft der Haute Couture - ein anderes, ergiebiges Analogon vorzuführen bestrebt ist: $\mathrm{St}$ il se $\mathrm{i}$, was $\mathrm{m}$ a $\mathrm{n}$ wolle, auch wenn der Produzent es anders meine als der Rezipient oder der Käufer, pardon!, der Händler.

Man werfe dieser Fehde gegen die herrschende - suizidäre - Verwechslung von Stil und Mode keinen $\gg$ Archaismus $\ll$ vor. Die $\gg$ radikale Subjektivität« ist nämlich so umwerfend neu nicht. Von Protagoras bis zur scholastischen Epistemologie, in welcher der Grundsatz der Prägung des Empfangenen durch das Empfangende galt: $\gg$ wie das Wasser die Form der Karaffe annimmt $\ll$ - doch schöpft der Frankenwein seinen Geist aus der Form des Bocksbeutels? -, wurde der Aspekt der $\gg$ Kreativität $\ll$ immer nur von Schulen vergessen, die nicht nur $\gg$ Stil $\ll$, sondern sogar $>$ Manier $\ll$ mit $>$ Nachahmung $<$ verwechselten, weil sie überhaupt die störende Relativität vergessen hatten ... Vielleicht hängt die Tragik des Revolutionären damit zusammen, daß es ursprünglich seine Kraft aus Zuwendung bezieht, sich aber seinen Weg von Abwendern diktieren läßt.

Dem Grammatiker sind allgemeine historische und philosophische Überlegungen wohl aufgezwungen, sind doch längst linguistische $\mathrm{Ge}$ meinplätze in philosophische Traktate und Manifeste aufgenommen worden, etwa das Axiom des 》arbitraire du signe«. Dabei ist es wie mit dem gespannten Gummiseil gegangen - ich meine die verbindliche $\gg$ Relativität《 bzw. die verbindenden $\gg$ Relationen $\ll-$, das über einen 
Abgrund an zwei Bäumen festgebunden war. Seit Jahrzehnten seufzen moderne und jetzt auch postmoderne Analytiker und Dekonstruktivisten über eine Relativität ohne Relation. Wann wieder verstanden wird, daB die Intelligenz nicht im Nichts das Höchste und Klügste und Schönste findet, weiB niemand. DaB aber das abgerissene Gummiseil nicht von alleine über den Abgrund auf das gegenüberliegende - und höhergelegene - Cañon-Ufer um den anderen Baum zurückschnellen wird, ahnt jeder. Genau, wie er weiß, daB wir über den Abgrund hinaus müssen und im Grunde auch wollen. Nur machen wir uns so ungern lächerlich ...

\section{$<$ Stil $>$ wie $<$ Stile $>=$ stetige Wahl}

Nun könnte man meinen, ich berufe mich auf ein geheimnisvolles, nicht falsifizierbares, angstlösendes zum Axiom erhobenes Als-ob-Postulat, daß der jenseits der Manier angesiedelte Stil nicht nur nicht mehr meßbar wäre, sondern mit der Ästhetik überhaupt nichts mehr zu tun hätte. Freilich wäre dann die These gesichert, daß nur dieser oder jener Stil meßbar ist, nicht aber Stil an und für sich! Die Ironie würde, natürlich unverbindlich, beipflichten: $\gg$ Stil überhaupt $\ll$ sei nicht meßbar, da er ja $>$ überhaupt kein Stik sei. Einen solchen Einwand würde ich nicht für unzulässig halten. Mehr noch, ich teile ihn gern, denn es liegt nicht in meinem Verständnis, daß man sich einfach ein Undefinierbares gibt, das in jedem Glasperlen-Spiel - mit Verlaub, stilzubrechen - zugleich als Libero und als Schiedsrichter eingesetzt werden dürfte.

Nicht nur von $\gg$ diesem oder jenem Stil $\ll$, sondern auch vom $\gg$ Stil überhaupt«, ist m.E. das doppelte, manchmal schwer verträgliche Hauptmerkmal die $\gg$ s t e t i g e W a h l «: Wahl zwischen vielen Möglichkeiten bzw. Varianten und zugleich kohärentes und konstantes, aufgeklärtes Verharren. Parodien und Plagiate werden meistens am Inhalt erkannt. D i e ser I n hal t is t a u c h Form. Er ist es sogar auf mehrfache Weise, was ich mit statutären Kategorien andeuten möchte:

1. Daß Inhalt auch Form ist, gilt zunächst für alles Rhematische. Der Inhalt der Prädikate ist <Konzept>, Bedeutung, in einem bestimmten Sinne also <subjektiv> bzw. <objektiv-subjektiv>.

2. DaB Inhalt auch Form ist, erhellt sich weiterhin aus dem Umstand, daB die bezeichnenden thematischen Koordinaten einen 'Lokalaugenschein' aus unserem <Perzept $>$ exzerpieren.

3. Die phematischen Brücken, die wir zwischen Konzept und Perzept schlagen, tragen noch stärker als das Rhematische und das Thematische, fast bis in die logischen Modalitäten hin, aber zweifellos in den epistemi- 
schen und den affektiven Modalitäten, das Merkmal des Ausgesuchten, Wählerischen, Persönlichen, Relativen.

4. SchlieBlich sind der jeden einzelnen Satz affizierende Weg des Argumentations-Schemas bzw. die Gangart des erzählenden oder darstellenden Textes wie jede Methode formale Angelegenheiten.

Wie bereits mehrfach erwähnt, halte ich diese vierfache Relativität für einen Aspekt der Objektivität, und zwar in doppelter Hinsicht: so ist Stil weder als kohärente und konstante Auswahl von Begriffen und Daten noch als Ausdruck der eigenen Freiheit $\gg$ gleichwertig $\ll$ bzw. $\gg$ gleichgültig《.

Deswegen ist $>$ Stil an sich - dem Stil-Begriff von Diderot und Goethe durchaus entsprechend - nicht meßbar. Denn, im Unterschied zu den doch ziemlich begrenzten ausdrucks-grammatischen, kasuellen, meßbaren Fügungen, sind die inhaltlich-formalen Potentialitäten wirklich 》unendlich $\ll$ oder mindestens $\gg$ unbegrenzt $\ll$. Den EntschluB, den Usus der Sprache auf die ästhetische $\gg$ Oberflächenstruktur $\ll z u$ beschränken, kann ich nicht nachvollziehen, denn auch die nicht meBbare Qualität des Stils hängt auch an seiner ästhetischen $\gg$ Tiefenstruktur $\ll$. Kurzum, <dieser oder jener Stil> verdient seinen Namen nur, wenn er auch $<$ Stil überhaupt $>$ ist. Ein Sophisma hieße nun, meßbar sei der Stil also nur, wenn er nicht meßbar ist! Aber Protagoras läßt sich auch anders verstehen. An der Schwelle von a posteriori und a priori-Urteilen, verstehe ich den Satz, $>$ der Mensch sei das Maß aller Dinge\&, nicht als Forderung einer $\gg$ subjektiven $\ll$ Relativität, sondern als Einsicht in die \objektive $\ll$ Relativität des Daseins, als Einsicht in seine rettenden Relationen.

$\gg$ Stil an und für sich $\ll$ ist weder meBbar noch kalkulierbar. Es stehen immer nicht nur unendlich viele Möglichkeiten ex parte objecti zur Wahl, sondern es gibt auch ex parte subjecti unendlich viele Wahlmöglichkeiten. [Mehr Möglichkeiten als der gute Stil hat wohl nur noch - freilich der schlechte Stil bzw. die Stillosigkeit.] Nichtsdestominder scheint mir in den kommenden Jahrzehnten eine Verbindung von Stilometrie und Lexikometrie den Bereich der Meßbarkeit über die Regularitäten der bloßen Manier hinaus erweitern zu können.

Dennoch dürfte niemals von der Angemessenheit auf die Einzigartigkeit geschlossen werden. Angenommen, jeder Teilnehmer dieser Jahrestagung über Stilfragen oder sonstige Leser dieser Zeilen müßte - problemgerecht, aber in beliebiger Länge und Form - darstellen, daß - um ein Beispiel zu nehmen, das uns Heutigen so bekannt ist wie es den Griechen in der Blütezeit der Akademie das Angeln war - die Stadt ursprünglich der 
Versuch des Menschen gewesen sei, sich den RAUM untertänig zu machen, und daB in vielen Städten und besonders in Vor- und Schlafstädten heute das Gegenteil eingetroffen sei, daf also die Stadt dem Menschen nicht Freiheit, sondern Sklaverei bringe, bestünden nicht mehr Möglichkeiten als Darsteller, denn jeder von uns würde sich überlegen, was er nun verfassen sollte: einen Artikel, eine Fabel, eine Epistel, ein Haiku, ein Manifest, ein mehrbändiges Geschichtsbuch, einen Dialog, eine Satire, eine Abhandlung, einen Roman, ein Drama, oder, was in diesem Falle den Grammatiker reizen dürfte, eine Utopie?

Eine andere, leichtere und dennoch vielgestaltige Aufgabe besteht in der angemessenen inventio und expositio, ohne circulus vitiosus, der nicht widersprüchlichen Thesen, d a $B$ dieser und jener Stil sehr wohl ziemlich meßbar ist, de $\mathrm{r} \mathrm{St}$ il a $\mathrm{n}$ i c h dage ge n let zten Endes überha upt $n$ ich t.

\section{Anhang}

Den Teilnehmern wurden die folgenden zwanzig Texte ausgeteilt, zu denen jeweils die Frage zu beantworten war, ob die Signatur stimmt (I) oder nicht (0). Von den am folgenden Tag eingereichten Antworten erreichte diejenige von Nicky Anders (Pseudonym von Marie-Anne Durand, Universität Grenoble) mit dreizehn richtigen I bzw. 0 die höchste Punktzahl und gewann damit eine Flasche Burgunder mit dem Prix d'excellence.

[Der Himmlische Lehrsaal]

Hätte ein Engel oder ein himmlischer Geist die Sprache erfunden: wie anders, als daß ihr ganzer Bau ein Abdruck von der Denkart dieses Geistes sein müßte; denn woran könnte ich ein Bild von einem Engel gemalt kennen als an dem Englischen, Überirdischen seiner Züge? Wo findet das aber bei unserer Sprache statt? Bau und Grundriß, ja selbst der ganze Grundstein dieses Palastes verrāt Menschenheit! In welcher Sprache sind himmlische, geistige Begriffe die ersten? Jene Begriffe, die auch nach der Ordnung unseres denkenden Geistes die ersten sein mūssen - Subjekte, notiones communes, die Samenkōrner unserer Erkenntnis, die Punkte, um die sich alles wendet und alles zurückführt, sind diese lebenden Punkte Elemente der Sprache? Die Subjekte müBten doch natürlicherweise vor dem Prädikat und die einfachsten Subjekte vor den zusammengesetzten, was da tut und handelt, das Wesentliche und Gewisse vor dem Ungewissen, Zufälligen vorhergegangen sein; - ja, was man nicht alles schließen könnte; - und in unsern ursprünglichen Sprachen findet durchgängig das offenbare Gegenteil statt. 
2 [Das eigentliche Grundgesetz]

Unsere Gesetze sind Versuche, aus Papier den weisen Mann zu machen, der allen Umständen gewachsen ist, und dessen Gerechtigkeit so groß ist wie seine Unerschrockenheit; - ach, wo ist das ehrfurchterweckende Gesicht des Gesetzgebers hin, welcher mehr bedeuten muß als das Gesetz, nämlich den Wunsch es aus Liebe und Ehrfurcht heilig zu halten?

Friedrich Nietzsche ??

3

[Der arme Dichter]

Ein Dichter, rund und feist bei Leibe,

Mit einem Antlitz', lang wie breit,

Und glänzend, wie des Vollmondes Scheibe,

Sprach einst von seiner Dürftigkeit,

Und schimpite brav auf theure Zeit.

$\gg$ Das thun Sie blob zum Zeitvertreibe,

Rief einer aus dem Kompanie;

Denn dieß Gedeihn an ihrem werthen Leibe,

Und ihr Gesicht, die schöne Vollmondscheibe,

Herr Kläger, zeugen wider Sie! «-

$\gg$ Das hat sich wohl! seufzt der Poet geduldig;

Doch, Gott gesegn'ihn! meinen Bauch -

Sanft strich er ihn - und diesen Vollmond auch -

Bin ich dem Speisewirth' noch schuldig. $\ll$

Gottfried August Bürger ??

[Der Unentbehrliche]

Könnt' ich tausendfach mich theilen,

Schnell mit allen Winden eilen,

Überall zugleich $\mathrm{zu}$ walten,

Wo's die Welt gilt zu gestalten!

Würden nicht durch meine Kräfte

Rasch gedeihn der Zeit Geschäfte?

Doch, so läßt mir mein Geschick

Schauen nur im Zeitungsblick

Ohne mich in fernen Reichen

Die verlassnen Völker schleichen! -

Von den Sternen möcht' ich wissen,

Ob sie mich nicht schwer vermissen?

Friedrich Rückert ??

5 [Die Mittagszeitung]

Korf erfindet eine Mittagszeitung, welche, wenn man sie gelesen hat, ist man satt. 
Ganz ohne Zubereitung irgendeiner andern Speise. Jeder auch nur etwas Weise hält das Blatt.

Christian Morgenstern ??

6 [Faust]

Was bist du denn? gier'ger Allumfasser

Des Universums kühner Freuer du.

Der blind, in Nacht, in zwiefach ew'gem Dunkel

Gebannt $2 u$ irren, nichts erkennen kannst,

Ein ewig ungelöstes Rätsel dir;

Erschaffer deiner Welt nach ewigen

Gesetzen, selbst von ihr erschaffen,

Was bist du mächt'ger, nicht'ger Erdenwurm?

Ein Gott in Banden, oder nur ein Staub?

Was ist des Denkens, was der Sinne Welt?

Die Zeit, der Raum, die Allumfassenden,

Und ihre Schöpfungen, durch die sie werden?

Was auBer ihnen, das Unendliche?

Was ist die Gottheit, jeder großen Kette

Ein erstes ewig unbegriffnes Glied,

Das, nicht getragen, alle Glieder trägt? -

Es wirft das Licht, das innre, dort hinaus

Auf ausgespannte Nacht die Bilder hin,

Ein leerer Widerschein des eignen Ichs,

Und so entsteht die Welt, die ich erkenne.

So hat - vielleicht der Zufall es geordnet,

Der große Bildner, den sie Gottheit nennen.

Und wenn, nicht bloß gedacht, dort Geist und Körper

Und Gottheit sind, - wie fass' ich sie? - umsonst!

Es treten ewig $\mathrm{z}$ wischen sie und mich

Der Sinne Lügen, der Vernunft Gesetze.

Theodor Körner ??

7 [Der Barfüßer]

Unterm Thor der Schenke lag

Trunken ein Barfüßer,

Von Gerichts- und Schöpfungstag

Sprach der fromme Büßer.

Sprach von Kaisern, Königen,

Fürsten, Grafen, Freien,

$\mathrm{DaB}$ sie seine frönigen

Unterthanen seien.

Und er ließ aus voller Hand

(Brauchte nicht zu sparen) 
Stāuben als wie Spreu und Sand

Kronen und Tiaren.

Und er gab das Diadem

Nur entschloßnen Trinkern,

Drohte mit dem Anathem

Allen matten Sinkern.

Über Bogen so und Bausch

Ordnet' er das Welttheil,

Und ihm war allein sein Rausch

Nicht ums Geld der Welt feil.

Nicolaus Lenau??

8 [Jenseits von Argument-Struktur und Rollenplan]

Auch Adverbiale stehen im Bannkreis des Verbs.

Judith Macheiner ??

9

[Entdeckung und Deduktion]

Um der Urbegriffe willen ist also noch zu bemerken: daß die Kategorien, als die wahren Stammbegriffe der reinen Vernunft, auch ihre eben so reinen abgeleiteten Begriffe haben, die in einem vollständigen System der Transzendental-Philosophie keineswegs übergangen werden können, mit deren bloßer Erwähnung aber ich in einem bloß kritischen Versuch zufrieden sein kann. Es sei mir erlaubt, diese reinen aber abgeleiteten Begriffe die Prādicamente (im Gegensatz der Prädikabilien) zu nennen. Wenn man die primitiven und ursprünglichen Begriffe hat, so lassen sich die subalternen und abgeleiteten leicht hinzufügen, und der Stammbaum der reinen Begriffe völlig ausmalen. Da es mir hier nicht um die Vollständigkeit des Systems, sondern nur der Principien zu einem System zu thun ist, so erspare ich die Ergänzung auf eine andere Beschäftigung. Man kann aber diese Absicht ziemlich erreichen, wenn man die Ontologischen Lehrbücher zur Hand nimmt, und z.B. der Kategorie der Causalitāt, die Prädikamente der Kraft, der Handlung, des Leidens, der der Gemeinschaft, die der Gegenwart, des Widerstandes, den Prädicabilien der Modalitāt, die des Entstehens, Vergehens, der Veränderung u.s.w. unterordnet. die Kategorien mit den modis der reinen Sinnlichkeit oder auch nur untereinander verbunden, geben eine große Menge abgeleiteter Begriffe und eine noch größere Menge unableitbarerer Begriffe, die zu verzeichnen eine nützliche, aber unerfüllbare, glücklicherweise aber zur Deduction der Urteilstafel entbehrliche Aufgabe wäre. Der Definition dieser Kategorien überhebe ich mich in dieser Abhandlung geflissentlich, ob ich gleich im Besitz dersellben sein möchte. Ich werde die Prädicabilien und die Prādicamente in der Folge bis auf den Grad zergliedern, welcher in Beziehung auf die Methodenlehre, die ich bearbeite, hinreichend ist. 
Ein Barde hieß, aus frommer Pflicht, Ein ganzes Heer von Sylben ringen.

Ich will nur $z$ wo zur Sprache $z$ wingen, Weil doch in Fabeln alles spricht.

Es sind die, so ich reden lasse, Machtwörter von der ersten Classe, Die in der Welt was rechtes schreyn, Die alten Feinde: Ja und Nein.

Es rüsten beide sich zum Streit.

Sie wollen nun als Helden fechten, Und nicht, wie kleine Hadrer, rechten. Kurz: sie bestimmen Ort und Zeit. Nein trotzt auf kriegerische Freude; $\mathrm{Ja}$ täuscht, verlockt, besticht die Feinde. Nein pocht auf Faustrecht und Gewalt; Ja traut auf seinen Hinterhalt.

Wir wollen, in gewissen Fällen, Uns beyde meisterlich verstellen, Am Hofe soll das Ja oft Nein, Und Nein ein wuchernd Jawort seyn.

Nein, das den Werth des Vorschlags sah, Beschloß, von nun an leeren $H a ̈ n d e n$ Den Beystand nimmer zu verpfänden, Und sprach zum erstenmale: Ja.

Die ganze Fehde war geschlichtet, Aus Eigennuz ein Bund erreichtet; Und beyde dienen izt der Welt, Nach Schweizerart, um baares Geld.

Friedrich von Hagedorn??

Was uns die ersten Silben freundlich nennen, Das ist dem Menschen wunderbar verwandt, Einst werden wir das Rätselbild erkennen, Von oben sonst den Vätern oft gesandt, Wenn sich die Seele wird vorn Körper trennen Und einzieh' $n$ in das alte Vaterland:

Da mag es freundlich, in der Jugend Prangen, Mit zarten Liebestōnen uns empfangen.

Die dritte Silbe baut sich auf der Erde Und ist dem Menschen immer wert und lieb; Und leichter trägt es seines Tags Beschwerde, Wenn's drin nur so froh und ohne Kummer blieb. Ach! wie so gern er zu ihm wiederkehrte, 
So ihn das Schicksal in die Ferne trieb;

So er hinaus muB in das wilde Leben,

Er scheidet still, doch bleibt er ihm ergeben.

Das Ganze prangt auf steilen Felsenhöhen

Als ein Vermächtnis der Vergangenheit;

Durch seine Mauern flüstert Geisterwehen

Wie stille Träume jener bessern Zeit.

Und wo hinaus die trunk'nen Blicke sehen,

Hat die Natur den Brautschmuck ausgestreut,

Als sollte hier die dritte Silbe prangen,

Die beiden ersten würdig zu empfangen.

Adalbert Chamisso??

[Monolog eines jungen Mannes von vierzig Jahren]

Das sind die Tage, über denen allen

Flamingoblaues Dämmern liegt.

- Und hören sehr perverse Orgeln schallen

Und Traueraffen aus den Bäumen fallen

Und sind sehr satt.

Und sind sehr matt und alt.

Und tragen uns mit kränklichen Gebärden

Und wissen, daß wir waren, was wir werden.

Und es ist gut.

Und schreiben ofter matte Operntexte.

Und zählen still vom ersten bis ins sechste.

Und sagen: "Wien".

Und sind noch immer wie vor $z$ wanzig Jahren.

Und wissen, daß wir werden, was wir waren.

Und wedeln sanft.

Und wandeln in der guten Abendröte.

Und spielen gern und sehr den alten Goethe.

Und sind sehr fein.

Und schreiben edle Auf- und Niedersätze.

Und stellen uns auf sehr belebte Plätze.

Und denken nichts.

Und haben einen sehr gepflegten Stil.

Und nicken mit dem Kopfe ganz und viel.

Und wirken ganz ornemental.

Und sind in dieser Welt wie in der Fremde.

Und tragen in der Nacht ein seidnes Hemde.

Und werden nächstens wohl katholisch werden. 
Der Muskel wird durch starken Gebrauch gestärkt, der Nerv hingegen dadurch geschwächt. Also übe man seine Muskeln durch jede angemessene Anstrengung, hūte hingegen die Nerven vor jeder, also die Augen vor zu hellem, besonders reflektiertem Lichte, von jeder Anstrengung in der Dämmerung, wie auch vor anhaltendem Betrachten zu kleiner Gegenstände, ebenso die Ohren vor zu starkem Geräusch, vorzüglich aber das Gehirn vor gezwungener, zu anhaltender oder unzeitiger Anstrengung; demnach lasse man es ruhen während der Verdauung, weil dann eben dieselbe Lebenskraft, welche im Gehirn Gedanken bildet, im Magen und in den Eingeweiden angestrengt arbeitet, Chymus und Chylus zu bereiten, ebenfalls während oder auch nach bedeutender Muskelanstrengung. Denn es verhält sich mit den motorischen wie mit den sensiblen Nerven, und wie der Schmerz, den wir in verletzten Gliedern empfinden, seinen wahren Sitz im Gehirn hat, so sind es auch eigentlich nicht die Beine und Arme, welche gehn und arbeiten, sondern das Gehirn, nämlich der Teil desselben, welcher mittelst des verlängerten und des Rückenmarks die Nerven jener Glieder erregt und dadurch diese in Bewegung setzt. Demgemäß hat auch die Ermūdung, welche wir in den Beinen oder Armen fühlen, ihren wahren Sitz im Gehirn, weshalb eben bloß die Muskeln ermūden, deren Bewegung willkürlich ist, d.h. vom Gehirn ausgeht, hingegen nicht die ohne Willkür arbeitenden wie das Herz. Offenbar also wird das Gehirn arbeiten wie das Herz. Offenbar also wird das Gehirn beeinträchtigt, wenn man ihm starke Muskeltätigkeit und geistige Anspannung zugleich oder auch nur dicht hintereinander abzwingt. Hiemit streitet es nicht, daß man im Anfang eines Spaziergangs oder überhaupt auf kurzen Gängen oft erhōhte Geistestātigkeit spürt; denn da ist noch keine Ermüdung besagter Gehirnteile eingetreten, und andererseits befördert eine solche leichte Muskeltätigkeit und die durch sie vermehrte Respiration das Aufsteigen des arteriellen, nunmehr auch besser oxydierten Blutes zum Gehirn.

Arthur Schopenhauer??

[Windgespräch]

Hast nie die Welt gesehn?

Hammerfest - Wien - Athen?

"Nein, ich kenne nur dies Tal, bin nur so ein Lokalwind -

kennst du Kuntzens Tanzsaal?"

Nein, Kind.

Servus! Muß davon!

Köln - Paris - Lissabon.

Christian Morgenstern ??

Der ergraute Mentor ungezāhlter Befragter, der Unermüdliche, dem es geglückt war, in zäher Bewußtheit die wenig wirtliche Landschaft sich gefügig zu ma- 
chen, der erfahrene Zuerkenner und väterlich gerechte Verteiler vorhandener Gelegenheit zu Rest und Erquickung, der Erzeuger endlich (und damit sind die Werke seiner Reifezeit kurz bezeichnet) jener aus Sahne bereiteten Alpenspeise, die unter dem Namen Käse Weltruf gewonnen hat: Peter Haslacher also, der Schutzhauswirt, rief der auf glatt gehobelten Schienen edel gewāhlten Holzes über die Schneefläche eilends Nähergleitenden eben noch ein warnendes Wort zu, als das Unheil schon seinen Lauf nahm. Erzeugt aus einer sanften Blendung des Auges in Verfolg der ungeahnt beschwingten Bewegung, erstanden aus einer leichten Berückung des Hirns, die das glückhafte Bewußtsein schwereloser Befreitheit auf nervlichen Bahnen zum rascher durchbluteten Herzen hinabtrug, aufgestiegen aus einer leichten Ermattung in jener Gegend des Knies, die ein nordischer Betrachter und durch ungekannt tiefe Vertrautheit gewichtiger Zeuge als die schönste des weiblichen Körpers zu bezeichnen sich nicht entbrechen zu sollen vermeint, hatte die Unsicherheit von den Schenkeln der Fahrtbeflissenen unversehens Besitz ergriffen, war höher kletternd in ungebührlich jäher Verbreitung über den unteren Leib bis in die Gegend des Zwerchfells gelangt und hatte den Schwerpunkt weit hinter die Linie lotrechten Standes zurück, ja die Wendende selbst bis zu schlechtweg unmittelbarer Berührung mit dem kristallisch weißen Elemente niedergedrängt: sie saß im Schnee.

Thomas Mann??

16

[Schiller]

Was wälzt sich dort mit Sprachgewalt

Von Pathos Tempel zur Tirade?

Ein Ungeheuer von Gestalt,

Das bald sich bãumet, bald sich ballt -

Hier wird Getümmel zur Ballade.

Wie schade.

Friedrich Torberg ??

17

Den Adler laß verschwenden

An Erben, Lamm und Frau

Das Salböl aus den Hānden

Des toten alten Pfau ...

Anonym ??

18 [Die Kristallschale]

Der Reichsfreiherr Eusebius horchte. Das Morgengrauen erhellte schon den westlichen Himmel. Es war kein Amselruf, erkannte der nun vollends erwachte Eusebius. Es kam aus der Kate drüben am Rande des Moores. War da ein Lebendiges in Bekümmernis, kreißte da eine werdende Mutter oder dergleichen? Sein war die Verantwortung für Alles.

Er eilte hinüber und fühlte sich erleichtert, als er nur ein etwa zweijähriges Mädchen antraf. Es war allein, er kannte es wohl, es war das Kind Krcyscz, 
die Mutter war wohl schon vor Morgengrauen aufgebrochen, um in der von Eusebius eingerichteten Sonnengoldspinnerei zu arbeiten, und einen Vater hatte es nie gehabt.

Das Kind blickte den Reichsfreiherrn Eusebius an, ganz ohne Scheu.

"Wie heißest du?" fragte er es.

"Pi", sagte es schlicht.

Pipistrella? dachte er leise. Die Fledermaus? Oder hat es auf der Wanderschaft wie so viele die Aussprache des Pi verlernt und heißt Philomela, die Nachtigall? Es wäre schön, wenn du Philomela hießest, fuhr er an das Kind hin zu denken fort.

Das Kind erriet seinen Gedanken sofort. „Glaubst du nicht auch, Herr Reichsfreiherr”, sagte es, „daß es noch ziemender für mich wäre, wenn ich die Lerche hieße statt der Nachtigall, da diese doch mehr der Liebenden Vogel ist?"

Er schickte sich an, einem wundersamen Erstaunen über die Seelenweisheit des Kindes nachzuhängen, aber eine zarte Unruhe nistete in seinem Herzen und ließ sich nicht mehr bannen. Pi, dachte er grübelnd, Pi? Erinnerte ihn nicht etwas an diesem Klange an seine eigene, unwiederbringlich vergangene Jugendzeit?

Ernst Wiechert??

[Dialektisch völlig verfahren]

Ich gebe der Genossin Seghers vollkommen recht in allem, was sie über die derzeitige Notwendigkeit der Herausstellung klassischer Formen sagt; aber stofflich liegt die Szene des verewigten Nationalpreisträgers falsch. Die propagandistische Linie ist korrekt, aber zu direkt. Wir müssen uns, ganz andere Methoden kopierend, viel langsamer heranpirschen. Vor allem ist zu bekämpfen die lächerliche westliche Vorstellung, daß wir (a) prüde und (b) humorlos seien. Das, in Kombination mit der von der Genossin mit Recht geforderten Form, kann am besten an einem völlig unpolitischen, dabei aber zugleich humoristischen und bourgeois-frivolen Thema unter Beweis gestellt werden. Ich habe mich daher entschlossen, die berüchtigten Verse der $\gg$ Wirtin an der Lahn $\ll$ in meine eigenen proletarisch-klassischen Metren umzudichten.

Stephan Hermlin??

[Das Gewissen und das Bewußtsein]

Die Antinomie der moralischen Weltanschauung, daß es ein moralisches Bewußtsein gibt und $d a \beta$ es keines gibt, - oder daß das Gelten der Pflicht ein Jenseits des Bewußtseins ist und umgekehrt nur in ihm stattfindet, war in der Vorstellung zusammengefaßt worden, worin das nichtmoralische Bewußtsein für moralisch gelte, sein zufälliges Wissen und Wollen für vollwichtig angenommen und die Glückseligkeit ihm aus Gnade zuteil werde. Diese sich selbst widersprechende Vorstellung nahm das moralische Selbstbewußtsein nicht über sich, sondern verlegte sie in ein ihm anderes Wesen. Aber dies Hinaussetzen dessen, was es als notwendig denken muß, außer sich selbst, ist ebenso der Widerspruch der Form nach, wie jener es dem Inhalte nach ist. Weil aber an sich eben das, was als widersprechend erscheint und in dessen Trennung und Wiederauflōsung die moralische Weltanschauung sich herumtreibt, desselbe ist, die reine Pflicht nämlich als das reine Wissen nichts anderes ist als das Sein des 
Bewußtseins, und das Selbst des Bewußtseins das Sein und die Wirklichkeit, ebenso was jenseits des wirklichen BewuBtseins sein soll, nichts anderes ist als das reine Denken, also in der Tat das Selbst ist, so geht für uns oder an sich das Selbstbewußtsein in sich zurück und weiß dasjenige Wesen als sich selbst, worin das Wirkliche zugleich reines Wissen und reine Pflicht ist. Es selbst ist sich das in seiner Zufäligkeit Vollgültige, das seine unmittelbare Einzelheit als das reine Wissen und Handeln, als die wahre Wirklichkeit und Harmonie.

Johann Gottlieb Fichte??

Für gewöhnlich enthalten satirisch gemeinte Parodien Elemente, meist inhaltlicher Art, an denen sie vom Leser erkannt werden sollen. Computer könnten es da wohl schwerer haben. Bei Fälschungen ist es umgekehrt: der Leser soll an der Echtheit des Textes nicht 2weifel. Möglicherweise hat es der mit Feinststatistik gespeiste Computer leichter, an der Echtheit des Schriftstückes Zweifel aufkommen zu lassen. Nichtsdestoweniger haben viele Autoren Texte verfaßt, die mit dem Bild nicht übereinstimmen, die andere Schriften von ihnen geben. Insbesondere sind verwechselte Autoren nicht leicht auseinanderzuhalten. Von epistemologisch großem Interesse ist indessen das Gefühl, das wohl jeder hat, sobald sich die Überraschung der Korrektur gelegt hat: a posteriori ist man nicht nur gescheiter, sondern meint auch, mit etwas mehr Aufmerksamkeit, Gedächtnis und List wäre man schon a priori nicht auf solche Vexierfragen hereingefallen.

[Lösung: 1, 1, 1, 0, 1, 0, 0, 1, 0, 1, 0, 0, 1, 1, 0, 1, 1, 0, 0, 0] 